\title{
Unlocking the Potential of High-Throughput Experimentation for Electrochemistry with a Standardized Microscale Reactor
}

\author{
Jonas Rein, James R. Annand, Michael K. Wismer, Jiantao Fu, Juno C. Siu, Artis Klapars, Neil A. \\ Strotman, Dipannita Kalyani*, Dan Lehnherr*, Song Lin*
}

\begin{abstract}
Organic electrochemistry has emerged as an enabling and sustainable technology in modern organic synthesis. Despite the recent renaissance of electrosynthesis, the broad adoption of electrochemistry in the synthetic community and, especially in industrial settings, has been hindered by the dearth of general, standardized platforms for high-throughput experimentation (HTE). Herein, we disclose the design of the HTe-Chem, a high-throughput microscale electrochemical reactor that is compatible with existing HTE infrastructure, and enables rapid evaluation of a broad array of electrochemical reaction parameters. Utilizing the $\mathrm{HTe}$ Chem to accelerate reaction optimization, reaction discovery, and chemical library synthesis is illustrated using a suite of oxidative and reductive transformations under constant current, constant voltage, and electrophotochemical conditions.
\end{abstract}

\section{Introduction}

Over the past decade, synthetic electrochemistry has garnered significant interest in the organic chemistry community. ${ }^{1-6}$ In an electrochemical reaction, electrons flowing between an anode and a cathode provide the redox equivalents to drive chemical transformations in lieu of traditional chemical oxidants or reductants. Under a sufficient current or potential, substrates can be oxidized or reduced at the electrode to generate reactive intermediates such as radicals and radical ions. The broad potential range of an electrochemical system allows for the activation of inert chemical bonds, thus driving highly endergonic reactions that are otherwise challenging or, sometimes, impossible. ${ }^{7}$ Meanwhile, by precisely dialing in a current or potential, chemists can select for a specific electron transfer reaction to occur in the presence of various reactive functional groups and do so with control over the reaction rate. These features make electrochemistry an attractive strategy for discovering fundamentally new chemical transformations and promoting green and efficient synthesis. ${ }^{1,3-5,7-9}$

A myriad of powerful electrochemical reactions developed in the past decade have shown the promise of electrochemistry as an enabling technology in both academic and industrial research. Despite the established advantages, electrochemical methods have seen only limited adoption in the pharmaceutical industry, primarily due to a lack of standardization and experimental flexibility. A lack of standardized electrolysis setups can render reaction reproduction challenging. ${ }^{10}$ To address this issue, standardized reactors for electrosynthesis have been designed and deployed, including an earlygeneration spatially addressable electrolysis platform (SAEP) ${ }^{11}$ and the recently commercialized ElectraSyn 2.012,13, e-Hive, and IKA Screening System ${ }^{14}$. These critical technology advancements have substantially accelerated the development and adoption of new electrosynthetic methods in the broader organic chemistry community. 2,15 Despite the success achieved with each of these systems, the ability to easily explore a broad array of electrochemical reaction parameters in a highthroughput fashion remains elusive and highly desirable. In this regard, an ideal standardized reactor platform should allow for convenient and modular programming of various experimental parameters. Although the multitude of reaction parameters present in electrochemical systems open opportunities for reaction discovery, they also pose unique challenges. For example, electrochemical parameters such as cell geometry, mode of operation (constant current or constant voltage), applied current or potential, electrode material, and electrolyte can be leveraged to improve reactivity. ${ }^{16}$ Nevertheless, this additional complexity introduces a barrier to begin investigating electrochemical reactions and also increases the number of conditions that must be evaluated. Thus, the full optimization can require significantly more screening experiments than traditional organic transformations. ${ }^{17}$

High-throughput experimentation (HTE) using standardized 24- or 96-well plates is routinely used for reaction discovery, optimization, and library synthesis in the pharmaceutical industry and beyond.18-21 The parallelization involved allows chemists to rapidly explore a large number of reaction parameters, generate a complete dataset, and optimize toward global maxima rather than local maxima, the latter of which are often encountered in traditional one-factor-at-a-time (OFAT) optimization. ${ }^{18,22}$ The adoption of standardized HTE systems, including parallel plate reactors, multichannel pipettors, liquid and solid handling robots, and stirring systems, has streamlined workflows by ensuring the compatibility of all equipment. This standardization also enables the use of preplated reagent libraries, which is particularly useful for the screening of catalysts, ligands, and reagents. ${ }^{18,19}$ Consequently, plate-based HTE, first introduced by the biology community in the 1950s, has today been expanded to virtually all research space in organic synthesis. $5,20,23-26$

The integration of HTE capabilities with organic electrosynthesis is needed to dramatically improve the 

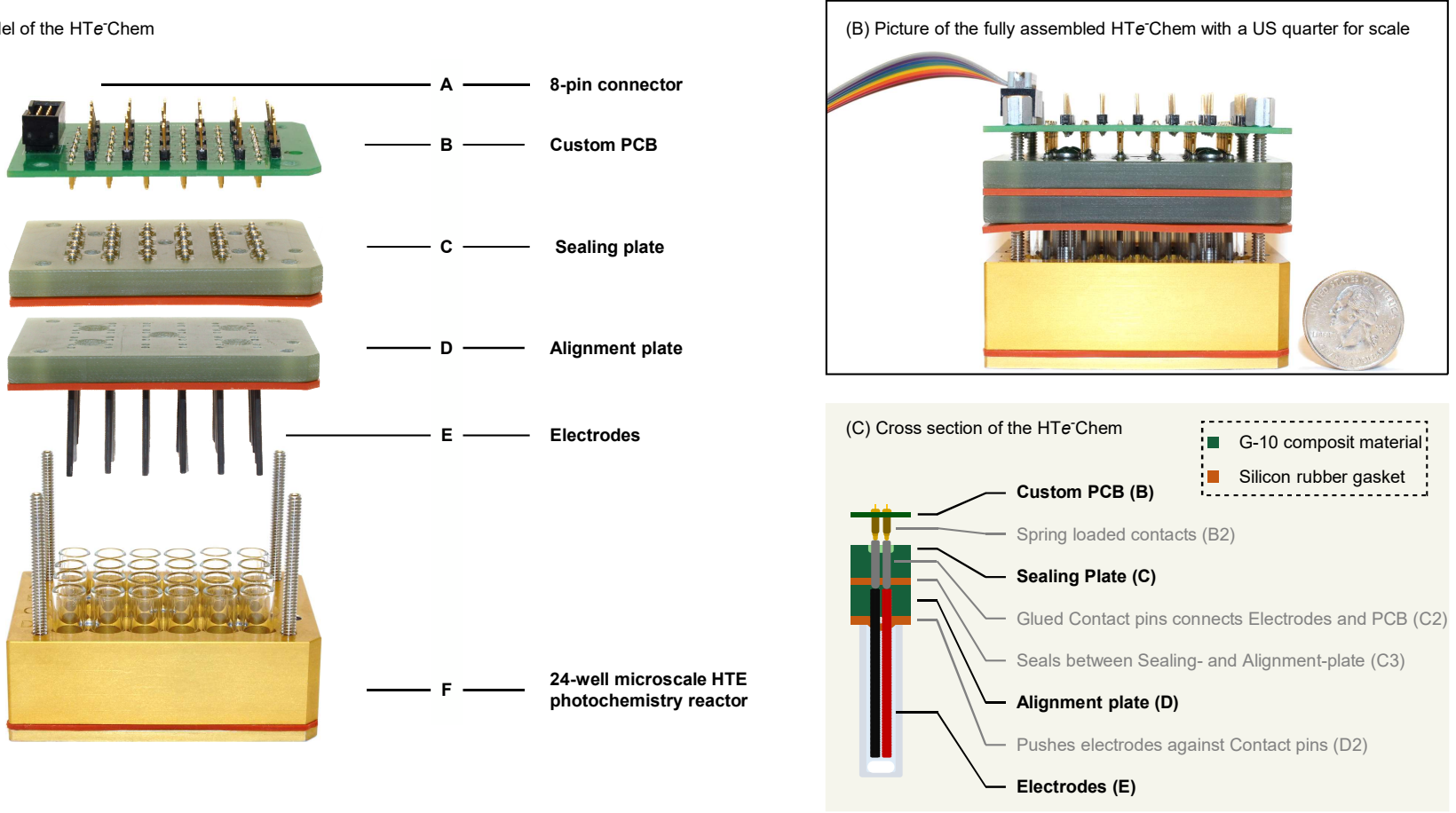

Figure 1: (A) Design and assembly of the HTe-Chem. (B) Picture of the fully assembled HTe-Chem with the constant potential top plate with a US quater for scale. (C) Cross section of the HTe-Chem.

adoption of electrochemistry in organic chemistry research. ${ }^{27}$ To this end, we aim to design an HTE platform that is compatible with the current HTE infrastructure and allows for the screening of all common electrochemical reaction parameters. An ideal HTE reactor for electrosynthesis will enable rapid screening of features unique to electrochemistry such as mode of operation (constant current or constant voltage), applied current or potential, applied charge, and electrode material. In addition, the reactor should be amenable to reactions at different temperatures and under air- and water-free conditions. Furthermore, the reactor will accommodate micro-scale reactions $(<400 \mu \mathrm{L}$ total reaction volume) to reduce material consumption, minimize cost, and improve safety. Finally, the screening platform should accommodate recent advancements such as electrophotochemistry 2,28 and alternating current electrolysis..$^{29,30}$ Notably, despite significant recent advances, a general, standardized HTE reactor that satisfies these desirable criteria remains elusive. ${ }^{31}$

Herein, we report the design of a 24-well plate high-throughput electrosynthesis reactor, namely $\mathrm{HT} e^{-C h e m}$, that presents all the aforementioned features and capabilities. By showcasing a selection of electrosynthetic transformations, we demonstrate the versatility of this new reactor for reaction discovery, optimization, and library synthesis in a variety of electrochemical applications.

\section{Design of HTe-Chem}

An important design principle for the $\mathrm{HT} e$-Chem reactor is that it needs to be based on a standardized commercial platform and should be operationally simple, modular, and chemically resistant. Our investigation commenced with a 24-well microscale HTE photochemistry reactor (F; Figure 1A), which features a 4 row by 6 column plate with $8 \times 30 \mathrm{~mm}$ glass vial inserts. This parent reactor has been extensively used in both academic and industrial settings for high-throughput screening experiments and is compatible with standardized equipment and existing infrastructure such as automated reagent addition systems and pre-plated reagent libraries. ${ }^{20,32}$ The reactor block has an insert for a temperature probe and can be heated or cooled using common laboratory temperature-control systems; it is also compatible with both magnetic rotary and tumble stirring. Finally, the base allows for efficient illumination of the vials with commercial HTE photochemistry equipment through the bottom of the reactor. Thus, this parent system provides an ideal platform for our design of a standardized and modular electrochemical HTE reactor.

The HTe-Chem (Figure 1) employs two parallel cylindrical rods with a diameter of $1.6 \mathrm{~mm}$ as the electrodes (E). Numerous conducting materials are commercially available in these dimensions in high purity, including graphite, nickel, stainless steel, copper, titanium, magnesium, zinc, platinum, tin, and aluminum. The electrodes are held closely apart at $1.54 \mathrm{~mm}$ distance (from surface to surface) and can perfectly fit in the microscale cells of the baseplate, resulting in a reaction volume (200-600 $\mu \mathrm{L})$ of up to 25 times lower than a typical electrochemical batch reactor. This compact arrangement reduces reagent usage and ensures sufficient conductivity at a low electrolyte concentration, while the cell geometry resembles that of a traditional batch cell and provides good scaling behavior. To keep the electrodes parallel, we designed an alignment plate (D) made from a chemically resistant and 
rigid G-10 composite material populated with holes machined with tight tolerances. In addition, a silicone rubber gasket (D2), which functions like a septum, is used to hold the electrodes in place and provides sealing against the glass vial inserts.

A sealing plate (C) made from the same G-10 composite material combined with an additional silicon rubber gasket (C3) was also included. This plate contains contact pins (C2) that are secured using epoxy resin and provide electrical contact between the printed circuit board (PCB; vide infra) and the electrodes while effectively excluding air and water from entering the system through the electrode-G10 contact. Upon assembly of the $\mathrm{HT} e^{-\mathrm{Chem}}$, the compression of the elastic silicon rubber gasket (D2) pushes the electrodes against the contact pins (C2). Even prolonged heating of the reactor does not result in significant loss of solvent, and the silicon rubber tolerates virtually any reaction conditions without damage or swelling. ${ }^{33}$ A detailed discussion of design decisions for the sealing and alignment top plates are outlined in the supporting information.

Separating the custom printed circuit board (B) from the electrode array increases the operational flexibility and the longevity of the system. Spring-loaded connectors (B2) on the PCB ensure good electrical contact with the contact pins (C2) on the sealing plate. Two variants of the PCB were designed to enable two distinct modes of operations, constant current electrolysis (CCE) and constant voltage electrolysis (CVE). An 8-pin connector (A) and a ribbon cable connect the PCB to four controllers, each of which supplies power to one row of the 24well matrix and allows for accurate control of current $( \pm 0.5 \%$ at $0.5 \mathrm{~mA})$ or cell voltage $( \pm 0.1 \%$ at $1.5 \mathrm{~V})$. This setup allows for the screening of 4 discrete currents or 4 discrete cell voltages depending on the mode of operation. One potential pitfall is that in the case of CCE, 6 cells are connected in series; hence, if one cell fails to conduct, electrolysis will fail in all connected cells. To address this issue, LEDs were added to each row as a visual identification of faulty cells. If a faulty cell is detected, our system allows the user to add a "skipper-pin" to the faulty cell to effectively disconnect it from the electric circuit. In addition, this feature allows for critical "no electricity" control experiments to be incorporated within the same experimental design, thus evaluating the necessity of applied current. Furthermore, users can start or stop a single reaction at any given time using the "skipper-pin" without affecting other reactions, which can help study the effect of the amount of applied charge on the reaction outcome. Therefore, this PCB design facilitates the flexible screening of up to 6 applied charges at 4 currents.

Importantly, the standardized plate design makes assembling the $\mathrm{HT}$-Chem reactor simple and straightforward. Most individual components are commercially available, including the HTE reactor block, silicon gaskets, electrodes, and power controllers. The components that are not yet commercially available, namely the alignment plate, sealing plate, and custom PCB, are readily manufactured and can be produced in bulk if needed. The complete technical drawings, specifications, troubleshooting advice, along with a video tutorial for the reactor setup are included in the SI.
The modular design of the $\mathrm{HT}$-Chem reactor allows for flexibility of reaction setup because the replacement of a single component is possible. For example, alternating current electrolysis can be easily implemented by substituting the power controllers for a waveform generator. In addition, electrophotochemical reactions can be readily carried out using an external, commercially available LED array (vide infra). Further, the design could, in principle, be extended to a 96-well plate format without major modifications. In the following section, we present the application of the $\mathrm{HT} e^{-\mathrm{Chem}}$ for a variety of known and new electrosynthetic transformations and demonstrate its exciting potential to accelerate the broader implementation of electrochemistry in the pharmaceutical industry.

\section{Applications}

We investigated the use of the HTe-Chem in a suite of oxidative and reductive transformations under constant current and constant voltage conditions, as well as in electrophotocatalytic systems. Potential use cases for reaction discovery, screening, and library synthesis were surveyed.

Oxidative Azidooxygenation Under Constant Current Conditions. We set out to demonstrate the reliability and reproducibility of the $\mathrm{HT} e$-Chem by quantifying the well-towell variability in a TEMPO-mediated azidooxygenation reaction previously reported by the Lin group. ${ }^{34,35}$ This model reaction was carried out in all 24 wells of the reactor under identical conditions using constant current electrolysis. Scaling down the reaction to the microscale $(17.5 \mu \mathrm{mol}, 380 \mu \mathrm{L})$ with the HTe-Chem furnished the difunctionalized styrene (2) with an average yield of $89.1 \%$, comparable to the $89 \%$ yield obtained in the original report under constant voltage conditions on a $0.2 \mathrm{mmol}$ scale (Scheme 1). The percent standard deviation was only $2.2 \%$, and there was no impact on the vial position even using a commonplace rotary stir plate. The reliability of the HTe-Chem reactor was further validated by researchers in three independent laboratories at Cornell University and Merck \& Co., Inc., Kenilworth, NJ, USA (see SI for details).

Scheme 1. Azidooxygenation of 4-methoxystyrene (1) adapted from constant voltage to constant current conditions on the HTeChem. The yields are determined by HPLC using dimethyl terephthalate as the internal standard.

Lin (2018):

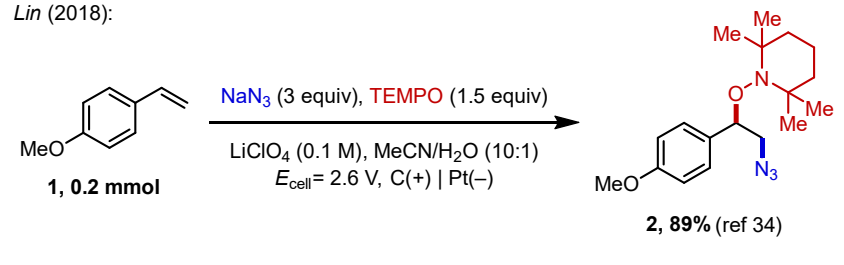

$17.5 \mu \mathrm{mo}$ $17.5 \mu \mathrm{mol}$
v), no supporting $0.6 \mathrm{~mA}, 2 \mathrm{~F}, \mathrm{C}(+) \mid \mathrm{Ni}(-)$ \% standard deviation: $2.2 \%$ 
Reductive Silylation Using A Sacrificial Anode. In many nonmediated electrochemical reactions, identifying the correct electrode material is key to rendering a reaction efficient and selective. ${ }^{36}$ As the unique surface chemistry influences the optimal electrochemical parameters, an ideal experimental design would evaluate various electrode materials with a range of current densities or applied voltages. The HTe-Chem presents precisely such capabilities, which we demonstrate in the further optimization of a reductive allylic silylation reaction recently developed in the Lin lab. ${ }^{37}$ By screening four currents with six cathode materials, we obtained the desired allylsilane (4) in a highest yield of $98 \%$ using platinum cathodes, representing a $23 \%$ increase over the original optimal $75 \%$ yield obtained on $1 \mathrm{mmol}$ scale with a graphite cathode (Figure 2). Intriguingly, use of stainless steel and platinum cathodes furnished the highest yields at 1.5-2.0 mA, whereas employment of graphite cathodes provided high yields at $0.5-$ 1.0 mA. These data highlight that factorial parallel screening allows the optimization toward a global maximum, while the traditional OFAT approach is prone to converging to local maxima (e.g. optimizing the current with a graphite cathode would result in the highest yield of $89 \%$ ). Furthermore, the successful demonstration of this reaction showcases the HTe Chem's compatibility with moisture sensitive, highly reducing conditions ( $-3.1 \mathrm{~V}$ vs. SCE or lower associated with the reduction of trimethylsilyl chloride ${ }^{37}$ ).

(A) Reaction conditions for the reductive synthesis of an allylsilane

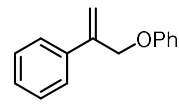

$3,17.5 \mu \mathrm{mol}$

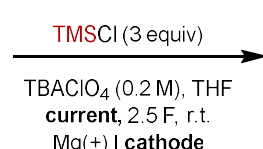

$\mathrm{Mg}(+) \mid$ cathode

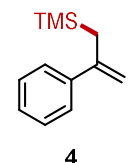

4
(B) Plate map and data for the reductive synthesis of an allylsilane

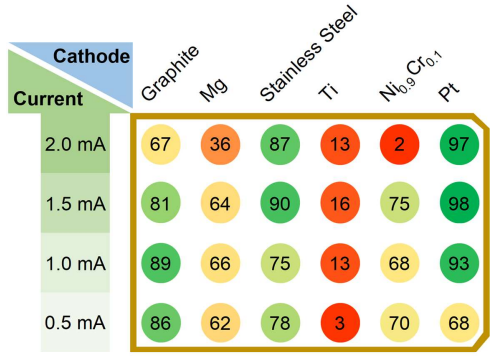

Figure 2. (A) Reaction conditions for the optimization of the reductive synthesis of allyl silanes. The reaction conditions that are varied on the plate are shown in bold. (B) Plate map and data for the optimization of the reductive synthesis of allyl silanes. The yields are determined by HPLC using 1,3,5-trimethoxy benzene as the internal standard.

Electrophotocatalysis Under Constant Voltage Conditions. Electrophotocatalysis has recently emerged as an enabling strategy for new reaction discovery. ${ }^{2}$ For example, Lambert et al. disclosed an elegant protocol for the oxidative $\mathrm{C}-\mathrm{H}$ amination of arenes by means of tandem electro- and photochemical activation of an organic catalyst (8). ${ }^{38}$ As the HTe-Chem's baseplate is designed to accommodate photochemistry, we aimed to leverage the HTe-Chem to optimize this electrophotochemical transformation. Thus, three cell voltages, three supporting electrolytes, and two different reaction times were surveyed. Furthermore, the capability of 24-well parallel screening allows crucial control experiments to be carried out simultaneously with reaction optimization under identical conditions. Accordingly, we included no electrolysis, no light, no electrolysis with no light, and no catalyst control reactions within the same plate (Figure $3)$. Using the HTe-Chem provided conditions resulting in a three-fold rate increase and furnished the desired product (7) in $78 \%$ yield after $24 \mathrm{~h}$, as compared to the originally reported $71 \%$ yield after $72 \mathrm{~h}$ on $0.4 \mathrm{mmol}$ scale, likely as a result of the improved light penetration and LED optical power. Notably, these reactions are also carried out under constant voltage electrolysis conditions, which is another important capability of the HTe-Chem reactor. As HTe-Chem is the first standardized platform for electrophotochemistry, we further established the reproducibility of the protocol. Setting up identical reactions in all 24 wells of the HTe-Chem gave an average yield of $75.0 \%$ with a percent standard deviation of $5.2 \%$ (see SI).

(A) Reaction condition for the electrophotochemical C-H amination of an arene.

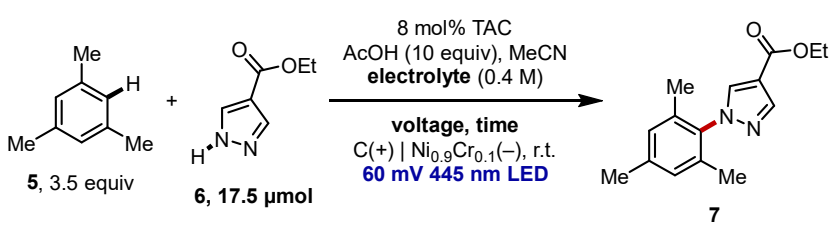

(B) Organocatalyst

(C) HTe-Chem electrophotochemistry setup
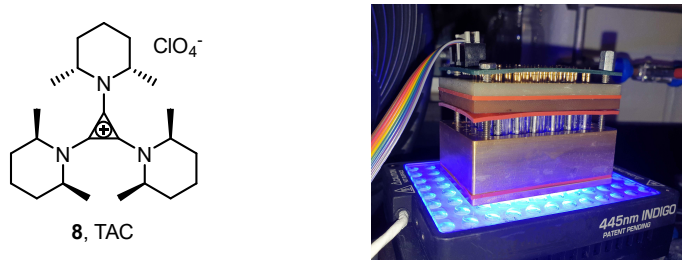

(D) Plate map and data of the electrophotochemical C-H amination of an arene.

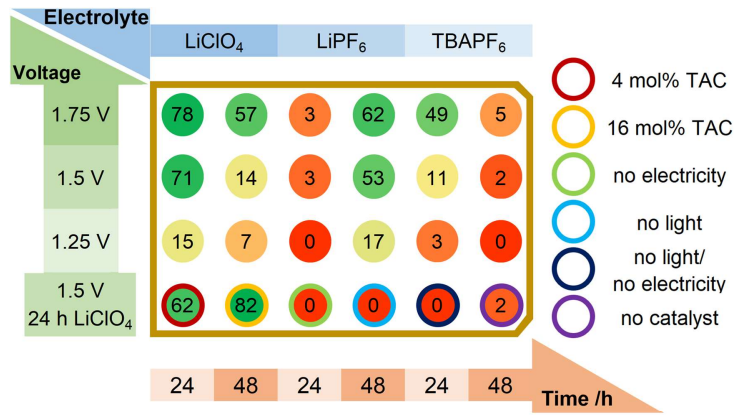

Figure 3. (A) Reaction conditions for the optimization of the oxidative $\mathrm{C}-\mathrm{H}$ amination of mesitylene (5) with pyrazol (6). The reaction conditions that are varied on the plate are shown in bold. (B) Trisaminocyclopropenium (TAC)(9) organocatalyst. (C) HTe-Chem while running the electrophotocatalytic reaction. The reactor is irradiated by the Lumidox II@ Gen II 24-position LED array at $445 \mathrm{~nm}$ with an optical power of $60 \mathrm{~mW}$ per vial. (D) 24-well plate data and experimental design. The yields are determined by HPLC using dimethyl terephthalate as the internal standard. 
Discovery and Optimization of the Chlorination of Arylboronic Acids. Building on the successful adaptation of a variety of electrochemical reactions to the HTe-Chem, we set out to optimize a previously unknown transformation, namely the electrochemical chlorination of boronic acids. We envisioned that the anodic oxidation of an arylboronic acid would give rise to an aryl radical, ${ }^{39}$ which could then be intercepted by an persistent chlorine atom source such as $\left[\mathrm{Mn}^{\mathrm{III}}\right]-\mathrm{Cl}$. This $\mathrm{Mn}^{\mathrm{III}}$ species has been employed in our previous work as an effective $\mathrm{Cl}$-atom transfer catalyst and can also be generated electrochemically from a $\mathrm{Mn}^{\mathrm{II}}$ salt. ${ }^{40}$ This methodology would be complementary to reports of electrochemical bromination and iodonation ${ }^{41}$ of boronic acids and provide a sustainable alternative to known methods using stoichiometric electrophilic chlorination reagents ${ }^{42,43}$.

The reaction was initially developed on a $0.2 \mathrm{mmol}$ scale using home-made electrolysis cells by subjecting 4-tert-butylphenyl boronic acid (12) to our previously reported electrochemical alkene dichlorination conditions (see SI for details). ${ }^{44}$ When the electrolysis was conducted at $65^{\circ} \mathrm{C}$, we detected a moderate yield $(\sim 30 \%)$ of the desired 4-tert-butylchlorobenzene (10) in addition to a significant amount of tert-butylbenzene (11) from unproductive protodeborylation. ${ }^{45}$ Subsequently, we employed the HTe-Chem to identify the design parameters that were important for observed reactivities with the goal of optimizing the chlorination reaction while minimizing the

(A) Reaction conditions for the chlorination of arylboronic acids

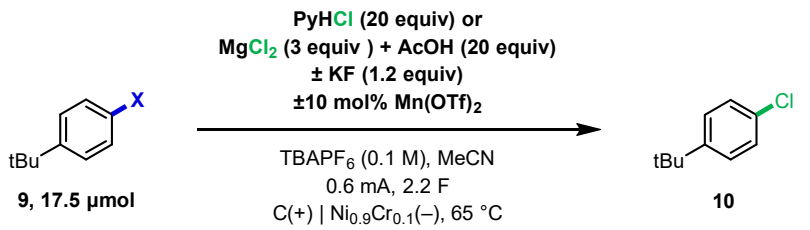

(B) Plate map and data for the optimzation with control reaction

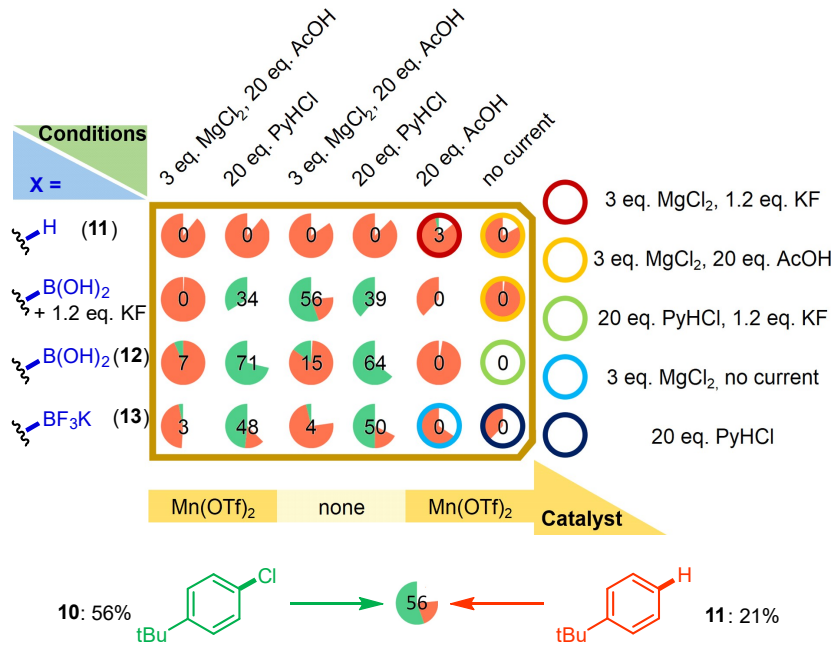

Figure 4. (A) Reaction conditions for the optimization of the electrochemical chlorination of aryl boronic acid (9). The reaction conditions that are varied on the plate are shown in bold. (B) Plate map with reaction yields of the desired 4-chlorotertbutylbenzene (10) (green) and of tert-butylbenzene (11) (red). The yields are determined by HPLC using dimethyl terephthalate as the internal standard. detrimental side reaction (Figure 4). In a single plate, the substrate and activating agent, chloride source, proton source, catalyst, and necessity of electrolysis were investigated. These data revealed several key insights into the reaction: (1) 11 is not an intermediate en route to product $\mathbf{1 0}$ (via electrophilic arene chlorination), (2) electricity is required to furnish the desired product, and (3) protodeborylation of the boronic acid occurs spontaneously with $\mathrm{AcOH}$ but not with the more weakly acidic pyridine $\cdot \mathrm{HCl}$. Notably, by using 4-tert-butylphenyl boronic acid (as opposed to $\mathrm{Ar}-\mathrm{BF}_{3} \mathrm{~K}$ ) as the substrate and pyridine hydrochloride as the acid and chlorine source, protodeborylation was fully suppressed, giving $71 \%$ of the desired product (10) in a procedure with manganese and a comparable $64 \%$ yield of $\mathbf{1 0}$ in metal-free conditions.

With these promising results, we carried out additional optimization of various other parameters including the catalyst (none, $\left.\mathrm{Mn}(\mathrm{OTf})_{2}, \mathrm{Ni}(\mathrm{OTf})_{2}, \mathrm{Fe}(\mathrm{OTf})_{2}\right)$, applied charges $(2 \mathrm{~F}, 3 \mathrm{~F}$, $4 \mathrm{~F})$, and loading of pyridine $\cdot \mathrm{HCl}(5,20$ equiv) on a single plate, which resulted in a further improved, metal-free protocol delivering quantitative yield (>99\%) of $\mathbf{1 0}$ (see SI for details). Scaling this chlorination to $0.2 \mathrm{mmol}$ scale furnished the same quantitative yield. These optimization data also showed that our initial mechanistic design employing a Mn catalyst is unnecessary, and that the electrophilic chlorinating agent can be generated directly via anodic oxidation. Finally, we note that the chlorination of boronic acids was rapidly developed through only two plates of screening experiments, thus significantly accelerating the optimization workflow vis-à-vis traditional screening strategies.

Electrochemical C-C Coupling Reactions. Various electrochemical $\mathrm{C}-\mathrm{C}$ coupling reactions have been reported recently, and adopting such transformations using the $\mathrm{HT} e$ Chem could enable rapid generation of diverse product libraries for medicinal chemistry studies. We successfully translated two such reactions, which were originally discovered using larger-scale commercial reactors, to the HTe Chem conditions (Scheme 2). For example, Baran and Minteer disclosed a reductive ketone-olefin coupling for the synthesis of medicinally relevant scaffolds. ${ }^{46}$ To adapt this method to the $\mathrm{HT} e^{-\mathrm{Ch}} \mathrm{em}$, we investigated a variety of cathode materials and applied currents and successfully replicated the reported results. Notably, the screening experiments allowed us to discovery a new optimal system using copper as the cathode, which was not examined in the original report and provided an improved 94\% yield in the synthesis of $\mathbf{1 6}$ (see SI for details).

In another report by Waldvogel and co-workers, an oxidative $\mathrm{C}-\mathrm{H} / \mathrm{C}-\mathrm{H}$ coupling was achieved to furnish valuable diarylmethanes 19 with hydrogen as the sole by-product. In an one-pot sequence, an electrochemically generated benzyl hexafluoroisopropyl ether $\mathbf{2 0}$ undergoes subsequent substitution with an electron-rich arene $\mathbf{1 8}$ in the presence of trifluoroacetic acid. ${ }^{47}$ This report showed that boron-doped diamond (BDD) electrodes promoted the optimal reactivity, generating product 19 in $93 \%$ yield on $1 \mathrm{mmol}$ scale. Interestingly, the $\mathrm{HT}$-Chem screening led us to further improve this methodology at a $17.5 \mu \mathrm{mol}$ scale and obtain the coupling product in quantitative yield with less expensive graphite electrodes (see SI for details). 
Baran and Minteer (2020) with Electrasyn 2.0:

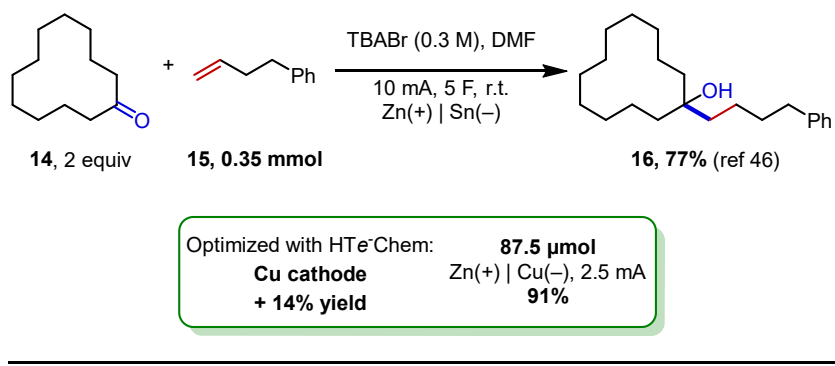

Waldvogel (2018) with IKA Screening System:
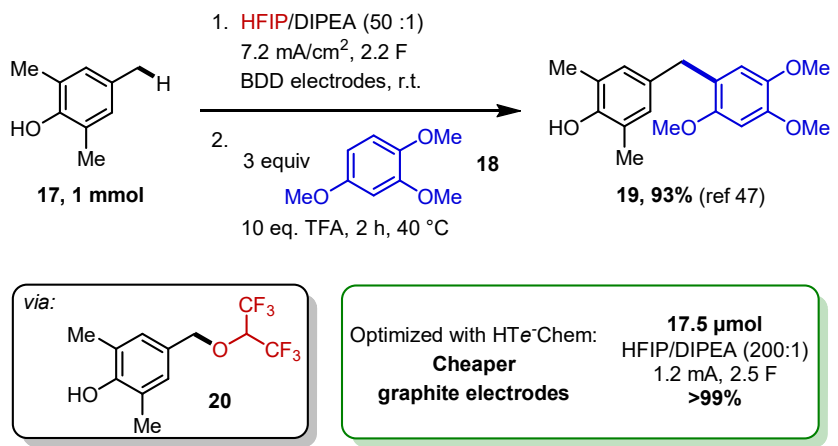

Scheme 2. Reductive and oxidative C-C cross coupling optimized using the HTe-Chem. The yields are determined by HPLC using 1,3,5-trimethoxybenzene for 16 and 4,4'-di-tert-butylbiphenyl for 19 as the internal standard.

Library synthesis. In addition to reaction development and optimization, use of the $\mathrm{HT} e$-Chem reactor offers the ability to rapidly generate a 24-membered library of compounds. This capability is of substantial interest to the pharmaceutical industry for parallel library synthesis using electrochemical methods. We demonstrated this capability through the synthesis of 24 hindered primary amines under reductive conditions via the coupling of iminium chlorides with cyanopyridines. ${ }^{15}$ To translate the originally reported procedure on $1 \mathrm{mmol}$ scale to $\mu \mathrm{mol}$ scale, the applied current

Lehnherr and Rovis (2019):

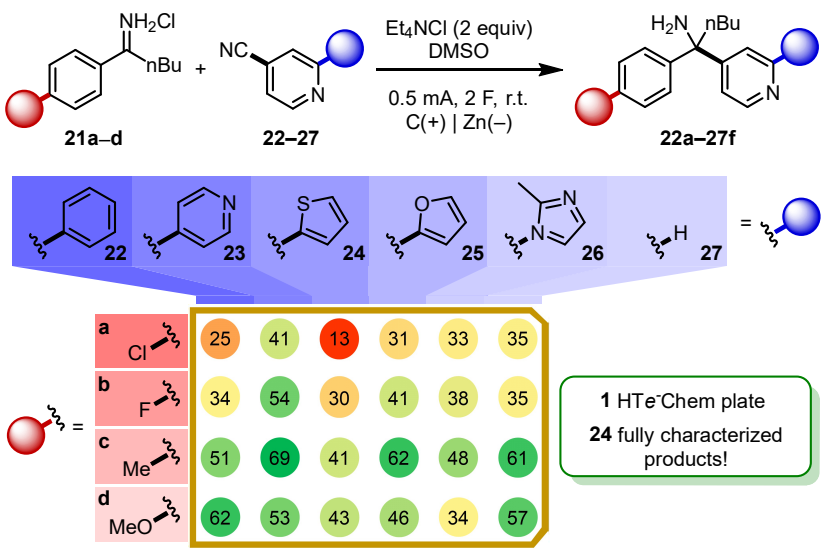

Figure 5. A reductive library synthesis of hindered amines. The yields shown in the plate map are LCAP. Calibrated yields determined by HPLC range from good to moderate (83-53\%) (See the SI for isolated yields and analytical details). was screened $(I=0.5,1.0,1.5$, and $2.0 \mathrm{~mA})$ using the HTe-Chem reactor which revealed that $0.5 \mathrm{~mA}$ with $2 \mathrm{~F}$ of charge was optimal for the coupling of 21c with 27 (see SI for details). These results are consistent with decreasing the current density to minimize the formation of diamine side-products. These optimal conditions were applied to library synthesis by reacting four iminium chlorides with six different cyanopyridines, providing rapid access to 24 primary amines with satisfactory isolated yields at $38.9 \mu \mathrm{mol}$ scale (Figure 5). Exploration of chemical space with the HTe-chem reactor enables observation of clear trends in the yields as the electronics of the iminium salt is varied, as illustrated in Figure 5 when comparing results down each column.

\section{Conclusion}

In sum, we report the design and application of the HTe-Chem, a high-throughput 24-well reactor for electrosynthesis. The $\mathrm{HT} e^{-\mathrm{Ch}}$ em was designed to enable parallel screening of electrochemically relevant parameters, such as electrode material, applied current or voltage, and electrolyte, as well as traditional reaction parameters, such as temperature, solvent, and catalyst. This reactor is based on a standardized commercial platform for HTE and is fully compatible with existing high-throughput infrastructure, including reagentadding equipment and pre-plated reagent libraries, and, as such, the design is expected to be scalable to 96 -well systems as well. We have demonstrated that employing this HTE reactor enables rapid optimization of reactions and is complementary to existing standardized electrochemistry systems, such as the ElectraSyn 2.0 and the IKA Screening System. In addition, the HTe-Chem accelerated the development of a previously unknown electrochemical reaction, with the optimal conditions readily scaled from the microscale of the reactor to typical benchtop scales. The HTe Chem is also compatible with cutting-edge developments in electrochemistry such as electrophotocatalysis. Finally, we show that the new reactor can be employed to rapidly generate chemical libraries relying upon electrochemical coupling reactions. Owing to the accessibility, flexibility, and performance of the HTe-Chem demonstrated here, we anticipate that this unprecedented access to a standardized platform for high-throughput experimentation in electrochemistry will substantially accelerate the adoption and development of electrochemical techniques both in academic and industrial settings.

\section{AUTHOR INFORMATION}

\section{Corresponding Authors}

Song Lin - Department of Chemistry and Chemical Biology, Cornell University, Ithaca, New York 14853, United States; orcid.org/0000-0002-8880-6476; Email: songlin@cornell.edu

Dan Lehnherr - Process Research and Development, Merck \& Co., Inc., Rahway, New Jersey, 07065, United States; Email: dan.lehnherr@merck.com

Dipannita Kalyani - Discovery Chemistry, Merck \& Co., Inc., Kenilworth, New Jersey, 07033, United States; Email: dipannita.kalyani@merck.com 


\section{Authors}

Jonas Rein - Department of Chemistry and Chemical Biology, Cornell University, Ithaca, New York 14853, United States; https://orcid.org/0000-0001-8237-6519

James R. Annand - Department of Chemistry and Chemical Biology, Cornell University, Ithaca, New York 14853, United States; https://orcid.org/0000-0002-2026-9985

Michael K. Wismer - Scientific Engineering and Design, Merck \& Co., Inc., Kenilworth, New Jersey, 07033, United States

Jiantao Fu - Discovery Chemistry, Merck \& Co., Inc., Kenilworth, New Jersey, 07033, United States

Juno C. Siu - Department of Chemistry and Chemical Biology, Cornell University, Ithaca, New York 14853, United States; https://orcid.org/0000-0003-4675-5399

Artis Klapars - Process Research and Development, Merck \& Co., Inc., Rahway, New Jersey, 07065, United States

Neil A. Strotman - Process Research and Development, Merck \& Co., Inc., Rahway, New Jersey, 07065, United States

Notes

The authors declare no competing financial interest.

\section{ACKNOWLEDGMENT}

Financial support was provided by NIGMS (R01GM130928) and Merck Sharp \& Dohme Corp., a subsidiary of Merck \& Co., Inc., Kenilworth, NJ, USA. This study made use of the NMR facility supported by the NSF (CHE-1531632). J.R. was supported by the ERP-Fellowship from the Studienstiftung des Deutschen Volkes. J.T. acknowledges support from the MRL Postdoctoral Fellowship program. We thank He Huang, Jeffery Galcznski for helpful discussions and constructive feedback; He Huang, Jeffery Galcznski, and Tristan Lambert for providing catalyst 8. We acknowledge the following at Merck, \& Co., Inc., Kenilworth, NJ, USA: Shane W. Krska for feedback and guidance with this project, Rebecca Ruck for feedback and manuscript editing, and May Ann Desaca for purification of products associated with the amine library synthesis.

\section{ABBREVIATIONS}

BDD, boron-doped diamond; CCE, constant current electrolysis; CVE, constant voltage electrolysis; HPLC, high performance liquid chromatography; HTE, high-throughput experimentation; LCAP, liquid chromatography area percent; LED, light-emitting diode; OFAT, one-factor-at-atime; TEMPO, (2,2,6,6-tetramethylpiperidin-1-yl)oxyl.

\section{References}

(1) Yan, M.; Kawamata, Y.; Baran, P. S. Synthetic Organic Electrochemical Methods since 2000: On the Verge of a Renaissance. Chem. Rev. 2017, 117 (21), 1323013319.

(2) Liu, J.; Lu, L.; Wood, D.; Lin, S. New Redox Strategies in Organic Synthesis by Means of Electrochemistry and Photochemistry. ACS Cent. Sci. 2020, 6 (8), 13171340.

(3) Möhle, S.; Zirbes, M.; Rodrigo, E.; Gieshoff, T.; Wiebe,
A.; Waldvogel, S. R. Modern Electrochemical Aspects for the Synthesis of Value-Added Organic Products. Angew. Chem. Int. Ed. 2018, 57 (21), 6018-6041.

Wiebe, A.; Gieshoff, T.; Möhle, S.; Rodrigo, E.; Zirbes, M.; Waldvogel, S. R. Electrifying Organic Synthesis. Angew. Chem. Int. Ed. 2018, 57 (20), 5594-5619.

(5) Frontana-Uribe, B. A.; Little, R. D.; Ibanez, J. G.; Palma, A.; Vasquez-Medrano, R. Organic Electrosynthesis: A Promising Green Methodology in Organic Chemistry. Green Chem. 2010, 12 (12), 2099-2119.

(6) Horn, E. J.; Rosen, B. R.; Baran, P. S. Synthetic Organic Electrochemistry: An Enabling and Innately Sustainable Method. ACS Cent. Sci. 2016, 2 (5), 302308.

(7) Manabe, S.; Wong, C. M.; Sevov, C. S. Direct and Scalable Electroreduction of Triphenylphosphine Oxide to Triphenylphosphine. J. Am. Chem. Soc. 2020, 142 (6), 3024-3031.

(8) Francke, R.; Little, R. D. Redox Catalysis in Organic Electrosynthesis: Basic Principles and Recent Developments. Chemical Society Reviews. Royal Society of Chemistry April 21, 2014, pp 2492-2521.

Song, L.; Fu, N.; Ernst, B. G.; Lee, W. H.; Frederick, M. O.; DiStasio, R. A.; Lin, S. Dual Electrocatalysis Enables Enantioselective Hydrocyanation of Conjugated Alkenes. Nat. Chem. 2020, 12 (8), 747-754.

(10) Beil, S.; Pollok, D.; Waldvogel, S. R. Reproducibility in Electro-organic Synthesis - Myths and Misunderstandings. Angew. Chem. Int. Ed. 2021, anie.202014544.

(11) Siu, T.; Li, W.; Yudin, A. K. Parallel Electrosynthesis of $\alpha$-Alkoxycarbamates, $\alpha$-Alkoxyamides, and $\alpha$ Alkoxysulfonamides Using the Spatially Addressable Electrolysis Platform (SAEP). J. Comb. Chem. 2000, 2 (5), 545-549.

(12) Yan, M.; Kawamata, Y.; Baran, P. S. Synthetic Organic Electrochemistry: Calling All Engineers. Angew. Chem. Int. Ed. 2018, 57 (16), 4149-4155.

Li, C.; Kawamata, Y.; Nakamura, H.; Vantourout, J. C.; Liu, Z.; Hou, Q.; Bao, D.; Starr, J. T.; Chen, J.; Yan, M.; Baran, P. S. Electrochemically Enabled, NickelCatalyzed Amination. Angew. Chem. Int. Ed. 2017, 56 (42), 13088-13093.

(14) Gütz, C.; Klöckner, B.; Waldvogel, S. R. Electrochemical Screening for Electroorganic Synthesis. Org. Process Res. Dev. 2016, 20 (1), 26-32.

(15) Lehnherr, D.; Lam, Y. H.; Nicastri, M. C.; Liu, J.; Newman, J. A.; Regalado, E. L.; Dirocco, D. A.; Rovis, T. Electrochemical Synthesis of Hindered Primary and Secondary Amines via Proton-Coupled Electron Transfer. J. Am. Chem. Soc. 2020, 142 (1), 468-478.

(16) Kingston, C.; Palkowitz, M. D.; Takahira, Y.; Vantourout, J. C.; Peters, B. K.; Kawamata, Y.; Baran, P. S. A Survival Guide for the "Electro-Curious." Acc. Chem. Res. 2020, 53 (1), 72-83. 
Xiang, J.; Shang, M.; Kawamata, Y.; Lundberg, H.; Reisberg, S. H.; Chen, M.; Mykhailiuk, P.; Beutner, G.; Collins, M. R.; Davies, A.; Del Bel, M.; Gallego, G. M.; Spangler, J. E.; Starr, J.; Yang, S.; Blackmond, D. G.; Baran, P. S. Hindered Dialkyl Ether Synthesis with Electrogenerated Carbocations. Nature 2019, 573 (7774), 398-402.

(18) Shevlin, M. Practical High-Throughput Experimentation for Chemists. Med. Chem. Lett. 2017, 8 (6), 601-607.

(19) Krska, S. W.; DiRocco, D. A.; Dreher, S. D.; Shevlin, M. The Evolution of Chemical High-Throughput Experimentation to Address Challenging Problems in Pharmaceutical Synthesis. Acc. Chem. Res. 2017, 50 (12), 2976-2985.

(20) Mennen, S. M.; Alhambra, C.; Allen, C. L.; Barberis, M.; Berritt, S.; Brandt, T. A.; Campbell, A. D.; Castañón, J.; Cherney, A. H.; Christensen, M.; Damon, D. B.; Eugenio De Diego, J.; García-Cerrada, S.; García-Losada, P.; Haro, R.; Janey, J.; Leitch, D. C.; Li, L.; Liu, F.; Lobben, P. C.; Macmillan, D. W. C.; Magano, J.; McInturff, E.; Monfette, S.; Post, R. J.; Schultz, D.; Sitter, B. J.; Stevens, J. M.; Strambeanu, I. I.; Twilton, J.; Wang, K.; Zajac, M. A. The Evolution of High-Throughput Experimentation in Pharmaceutical Development and Perspectives on the Future. Org. Process Res. Dev. 2019, 23 (6), 1213-1242.

(21) Welch, C. J. High Throughput Analysis Enables High Throughput Experimentation in Pharmaceutical Process Research. React. Chem. Eng. 2019, 4 (11), 1895-1911.

(22) Allen, C. L.; Leitch, D. C.; Anson, M. S.; Zajac, M. A. The Power and Accessibility of High-Throughput Methods for Catalysis Research. Nat. Catal. 2019, 2 (1), 2-4.

(23) Shultz, C. S.; Krska, S. W. Unlocking the Potential of Asymmetric Hydrogenation at Merck. Acc. Chem. Res. 2007, 40 (12), 1320-1326.

(24) Burgess, K.; Lim, H. J.; Porte, A. M.; Sulikowski, G. A. New Catalysts and Conditions for a C-H Insertion Reaction Identified by High Throughput Catalyst Screening. Angew. Chem. Int. Ed. 1996, 35 (2), 220222.

(25) McNally, A.; Prier, C. K.; MacMillan, D. W. C. Discovery of an $\alpha$-Amino $\mathrm{C}$-H Arylation Reaction Using the Strategy of Accelerated Serendipity. Science. 2011, 334 (6059), 1114-1117.

(26) Santanilla, A. B.; Regalado, E. L.; Pereira, T.; Shevlin, M.; Bateman, K.; Campeau, L. C.; Schneeweis, J.; Berritt, S.; Shi, Z. C.; Nantermet, P.; Liu, Y.; Helmy, R.; Welch, C. J.; Vachal, P.; Davies, I. W.; Cernak, T.; Dreher, S. D. Nanomole-Scale High-Throughput Chemistry for the Synthesis of Complex Molecules. Science. 2015, 347 (6217), 49-53.

(27) HTE systems for the testing of battery materials have been developed and used in battery research but are not suitable for electrosynthesis applications. See: Hahn, R.; Ferch, M.; Tribowski, K.; Kyeremateng, N. A.; Hoeppner, K.; Marquardt, K.; Lang, K. D.; Bock, W.
High-Throughput Battery Materials Testing Based on Test Cell Arrays and Dispense/Jet Printed Electrodes. Microsyst. Technol. 2019, 25 (4), 1137-1149.

(28) Yu, Y.; Guo, P.; Zhong, J. S.; Yuan, Y.; Ye, K. Y. Merging Photochemistry with Electrochemistry in Organic Synthesis. Org. Chem. Front. 2019, 7 (1), 131-135.

Rodrigo, S.; Um, C.; Mixdorf, J. C.; Gunasekera, D.; Nguyen, H. M.; Luo, L. Alternating Current Electrolysis for Organic Electrosynthesis: Trifluoromethylation of (Hetero)Arenes. Org. Lett. 2020, 22 (17), 6719-6723.

(30) Bortnikov, E. O.; Semenov, S. N. Coupling of Alternating Current to Transition-Metal Catalysis: Examples of Nickel-Catalyzed Cross-Coupling. J. Org. Chem. 2021, 86, 782-793.

(31) Jensen disclosed a platfrom for screening of electrosynthesis and analytical electrochemsitry under flow chemsitry conditions. See: Mo, Y.; Rughoobur, G.; Nambiar, A. M. K.; Zhang, K.; Jensen, K. F. A Multifunctional Microfluidic Platform for HighThroughput Experimentation of Electroorganic Chemistry. Angew. Chem. Int. Ed. 2020, 59 (47), $20890-20894$.

Shevlin, M.; Friedfeld, M. R.; Sheng, H.; Pierson, N. A.; Hoyt, J. M.; Campeau, L. C.; Chirik, P. J. NickelCatalyzed Asymmetric Alkene Hydrogenation of $\alpha, \beta$ Unsaturated Esters: High-Throughput Experimentation-Enabled Reaction Discovery, Optimization, and Mechanistic Elucidation. J. Am. Chem. Soc. 2016, 138 (10), 3562-3569.

See SI for Harsh Chemcical Conditions That Might Not Be Fully Compatible with Our System.

Siu, J. C.; Sauer, G. S.; Saha, A.; MacEy, R. L.; Fu, N.; Chauviré, T.; Lancaster, K. M.; Lin, S. Electrochemical Azidooxygenation of Alkenes Mediated by a TEMPON3 Charge-Transfer Complex. J. Am. Chem. Soc. 2018, 140 (39), 12511-12520.

(35) Preliminary Data Suggested That the Azide Loading Could Be Reduced to 1.5 Equivalents under Constant Current Conditions.

(36) Heard, D. M.; Lennox, A. J. J. Electrode Materials in Modern Organic Electrochemistry. Angew. Chem. Int. Ed. 2020, 132 (43), 19026-19044.

Lu, L.; Siu, J. C.; Lai, Y.; Lin, S. An Electroreductive Approach to Radical Silylation via the Activation of Strong Si-Cl Bond. J. Am. Chem. Soc. 2020, 142, 2127221278.

(38) Huang, H.; Strater, Z. M.; Rauch, M.; Shee, J.; Sisto, T. J.; Nuckolls, C.; Lambert, T. H. Electrophotocatalysis with a Trisaminocyclopropenium Radical Dication. Angew. Chem. Int. Ed. 2019, 58 (38), 13318-13322.

(39) Yan, G.; Yang, M.; Wu, X. Synthetic Applications of Arylboronic Acid via an Aryl Radical Transfer Pathway. Org. Biomol. Chem. 2013, 11 (46), 79998008.

(40) Ye, K. Y.; Pombar, G.; Fu, N.; Sauer, G. S.; Keresztes, I.; Lin, S. Anodically Coupled Electrolysis for the 
Heterodifunctionalization of Alkenes. J. Am. Chem. Soc. 2018, 140 (7), 2438-2441.

(41) Fu, Z.; Hao, G.; Fu, Y.; He, D.; Tuo, X.; Guo, S.; Cai, H. Transition Metal-Free Electrocatalytic Halodeborylation of Arylboronic Acids with Metal Halides MX (X = I, Br) to Synthesize Aryl Halides. Org. Chem. Front. 2020, 7 (3), 590-595.

(42) Wu, H.; Hynes, J. Copper-Catalyzed Chlorination of Functionalized Arylboronic Acids † . Org. Lett. 2010, 12 (6), 1192-1195.

(43) Szumigala, R. H.; Devine, P. N.; Gauthier, D. R.; Volante, R. P. Facile Synthesis of 2-Bromo-3Fluorobenzonitrile: An Application and Study of the Halodeboronation of Aryl Boronic Acids. J. Org. Chem. 2004, 69 (2), 566-569.

(44) Fu, N.; Sauer, G. S.; Lin, S. Electrocatalytic Radical Dichlorination of Alkenes with Nucleophilic Chlorine Sources. J. Am. Chem. Soc. 2017, 139 (43), 1554815553.

(45) Kuivila, H. G.; Nahabedian, K. V. Electrophilic Displacement Reactions, X. General Acid Catalysis in the Protodeboronation of Areneboronic Acids. J. Am. Chem. Soc. 1961, 83 (9), 2159-2163.

(46) Hu, P.; Peters, B. K.; Malapit, C. A.; Vantourout, J. C.; Wang, P.; Li, J.; Mele, L.; Echeverria, P. G.; Minteer, S. D.; Baran, P. S. Electroreductive Olefin-Ketone Coupling. J. Am. Chem. Soc. 2020.

(47) Imada, Y.; Röckl, J. L.; Wiebe, A.; Gieshoff, T.; Schollmeyer, D.; Chiba, K.; Franke, R.; Waldvogel, S. R. Metal- and Reagent-Free Dehydrogenative Formal Benzyl-Aryl Cross-Coupling by Anodic Activation in 1,1,1,3,3,3-Hexafluoropropan-2-Ol. Angew. Chem. Int. Ed. 2018, 57 (37), 12136-12140. 